Received: 21 March 2017

Accepted: 7 June 2017

Published online: 17 July 2017

\section{OPEN The Epichloë festucae antifungal protein has activity against the plant pathogen Sclerotinia homoeocarpa, the causal agent of dollar spot disease}

\author{
Zipeng Tian ${ }^{1}$, Ruying Wang ${ }^{1}$, Karen V. Ambrose ${ }^{1,2}$, Bruce B. Clarke ${ }^{1}$ \& Faith C. Belanger ${ }^{1}$
}

Epichloë spp. are naturally occurring fungal endophytic symbionts of many cool-season grasses. Infection by the fungal endophytes often confers biotic and abiotic stress tolerance to their hosts. Endophyte-mediated disease resistance is well-established in the fine fescue grass Festuca rubra subsp. rubra (strong creeping red fescue) infected with E. festucae. Resistance to fungal pathogens is not an established effect of endophyte infection of other grass species, and may therefore be unique to the fine fescues. The underlying mechanism of the disease resistance is unknown. E. festucae produces a secreted antifungal protein that is highly expressed in the infected plant tissues and may therefore be involved in the disease resistance. Most Epichloë spp. do not have a gene for a similar antifungal protein. Here we report the characterization of the E. festucae antifungal protein, designated Efe-AfpA. The antifungal protein partially purified from the apoplastic proteins of endophyte-infected plant tissue and the recombinant protein expressed in the yeast Pichia pastoris was found to have activity against the important plant pathogen Sclerotinia homoeocarpa. Efe-AfpA may therefore be a component of the disease resistance seen in endophyte-infected strong creeping red fescue.

Epichloë spp. are common naturally occurring fungal endophytic symbionts of many cool-season grasses ${ }^{1,2}$. The fungi are systemic within the above-ground portions of the plant. They grow exclusively in between the plant cells through a novel intercalary hyphal extension process ${ }^{3}$. Infection by the fungal endophytes often confers biotic and abiotic stress tolerance to their hosts ${ }^{4}$. Resistance to insect and mammalian herbivory has been associated with the synthesis of toxic alkaloids by the fungal endophytes ${ }^{5}$. In some endophyte-plant associations, drought tolerance and disease resistance have also been attributed to infection by fungal endophytes, but the underlying mechanisms are not fully understood ${ }^{6-9}$.

The fine fescues strong creeping red fescue (Festuca rubra subsp. rubra) and Chewings fescue (Festuca rubra subsp. commutata) are commercially important low-maintenance turfgrasses ${ }^{10}$. The main location of seed production in the United States is Oregon. Over 18 million pounds of Festuca rubra seed were produced in Oregon in 2015 (http://cropandsoil.oregonstate.edu/system/files/u1473/GrassSeedProduction2015.pdf).

Endophyte-infected cultivars are generally preferred because of their enhanced insect, stress, and disease resistance. Endophyte-mediated disease resistance to the fungal pathogens Laetisaria fuciformis (McAlp.) Burdsall, the causal agent of red thread disease, and Sclerotinia homoeocarpa Bennett, the causal agent of dollar spot disease, has been well established in the fine fescues ${ }^{6,7}$. Both diseases are serious management problems for Festuca rubra ${ }^{7,11}$. Current breeding efforts include incorporation of E. festucae into new cultivars to reduce the necessity for fungicide use for disease control ${ }^{7,12}$.

Disease resistance as observed in the endophyte-infected fine fescues is not a general feature of endophyte-infected grasses ${ }^{13}$. No such endophyte-mediated Sclerotinia homoeocarpa resistance was seen in perennial ryegrass (Lolium perenne L.) field trials ${ }^{7}$. Similarly, no endophyte-mediated resistance to brown

${ }^{1}$ Department of Plant Biology, Rutgers University, New Brunswick, New Jersey, 08901, USA. 2Present address: Indigo Agriculture, Charlestown, Massachusetts, 02129, USA. Correspondence and requests for materials should be addressed to F.C.B. (email: belanger@aesop.rutgers.edu) 
Leaf

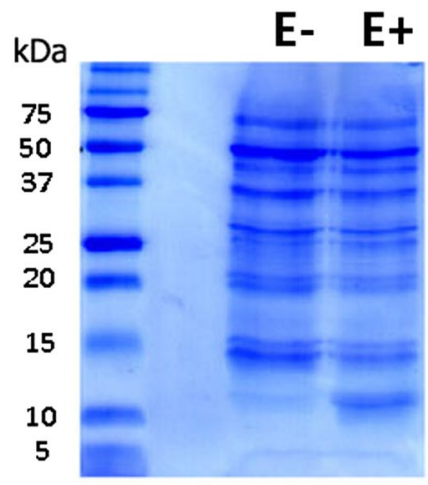

\section{Sheath}

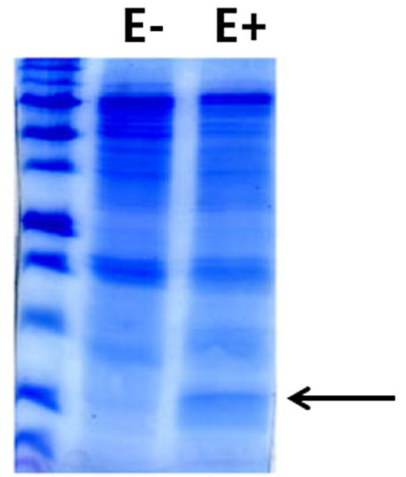

Figure 1. SDS-PAGE analysis of apoplastic proteins from leaf and leaf sheath tissue of endophyte-free (E-) and endophyte-infected $(\mathrm{E}+)$ plants. Protein standards molecular masses in $\mathrm{kDa}$ are indicated on the left side of the figure. The arrow indicates the band cut for protein sequence analysis.

patch caused by Rhizoctonia solani Kuhn or to Puccinia graminis subsp. graminicola was observed in tall fescue (Schedonorus arundinaceus (Schreb.) Dumont. = Lolium arundinaceum (Schreb.) Darbysh., formerly Festuca arundinacea Schreb. $)^{14,15}$. Overall, breeders have not reported endophyte-mediated resistance to fungal pathogens in the cultivated grasses perennial ryegrass or tall fescue.

The literature on effects of Epichloë endophyte infection on disease resistance of various host grasses is conflicting. Hume et al. ${ }^{16}$ present a comprehensive summary of some of the conflicting results. Endophyte-mediated disease resistance may therefore be unique to the fine fescues since field-level disease resistance has not been conclusively established in other Epichloë-grass associations.

In a quantitative transcriptome study of the Epichloë festucae-strong creeping red fescue interaction ${ }^{17}$, the second most abundant fungal transcript, representing $6 \%$ of the fungal transcripts in planta, encoded a protein similar to antifungal proteins from Penicillium and Aspergillus species ${ }^{18}$. Based on the activity assays described below for the E. festucae protein, the designations of similar genes from other fungal species, and nomenclature recommendations for Epichloë spp. ${ }^{19}$, the E. festucae gene is designated Efe-afpA and the protein is designated $E f e$-AfpA. The antifungal protein gene found in E. festucae infecting strong creeping red fescue is not present in most Epichloë genomes for which whole genome sequence is available ${ }^{17}$. The transcript abundance and the limited existence of the gene among Epichloë spp. suggested the E. festucae antifungal protein may be a component of the unique endophyte-mediated disease resistance observed in strong creeping red fescue. Here we report the functional characterization of the E. festucae antifungal protein. The purified recombinant antifungal protein was found to have activity against the important turfgrass fungal pathogen Sclerotinia homoeocarpa, the causal agent of dollar spot disease.

\section{Results}

The $E$. festucae Antifungal Protein is a Component of the Apoplastic Proteins from Infected Strong Creeping Red Fescue. The E. festucae antifungal protein Efe-AfpA (gene model EfM3.063660) was predicted to be a secreted protein by the program Target $\mathrm{P}^{17,20}$. As a secreted protein, it was expected to be in the apoplastic fluid of the infected plant tissue. Therefore the apoplastic proteins were isolated from leaves and leaf sheaths from endophyte-free and endophyte-infected plants and compared on an SDS-polyacrylamide gel (Fig. 1). A band at about $10 \mathrm{kDa}$ was more prominent in both the leaf and leaf sheath samples from the endophyte-infected plant than in the samples from the endophyte-free plant.

The $10 \mathrm{kDa}$ band was excised from the endophyte-infected leaf sheath sample and subjected to LC/MS/MS peptide sequencing. The E. festucae antifungal protein was the most abundant protein detected in the sample, confirming that it is indeed a secreted protein. Numerous other E. festucae proteins were also detected at lower abundance and information on those proteins is presented in Supplementary Table S1. No plant proteins were detected, likely because there is limited sequence information available for the host, strong creeping red fescue.

Protein Sequence Comparisons of the E. festucae Antifungal Protein with Similar Antifungal Proteins. The E. festucae antifungal protein sequence is similar to proteins from several other fungal species from the subphylum Pezizomycotina ${ }^{17}$. The most well-characterized are designated AFP (antifungal protein) from Aspergillus giganteus ${ }^{21,22}$, NFAFP from Neosartorya fischeri (synonym Aspergillus fischeri) ${ }^{23}$ and two similar yet distinct proteins, PAF and PgAFP, from Penicillium chrysogenum ${ }^{24-26}$. A comparison of these protein sequences with the Efe-AfpA sequence is shown in Fig. 2. Phylogenetic analysis of the amino acid sequences indicates the Efe-AfpA sequence is more similar to PAF than to the other antifungal protein sequences in the comparison (Supplementary Fig. S1), as was reported previously for the DNA coding sequences ${ }^{17}$. All of these characterized proteins are known to be secreted and the predicted signal peptide cleavage sites are indicated in Fig. 2. The experimentally determined N-termini of the similar proteins are indicated in Fig. 2. In all cases it is 


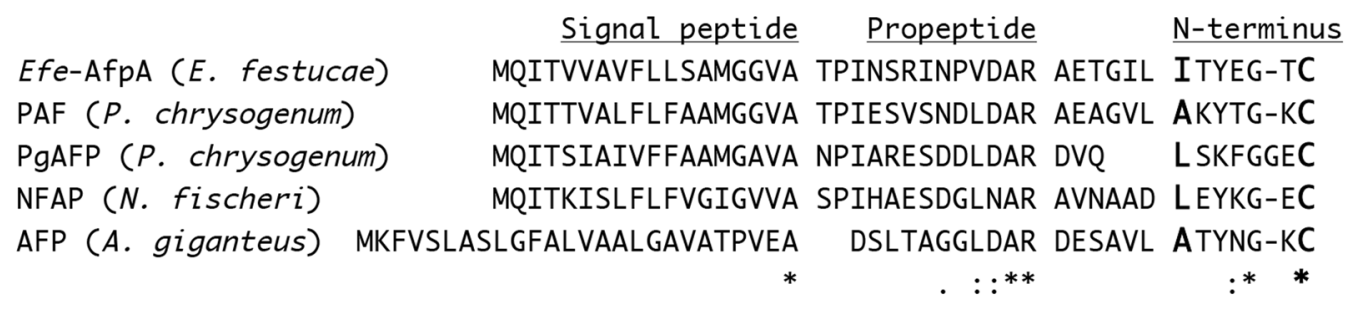

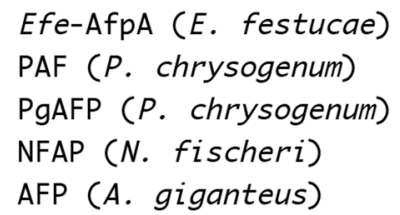

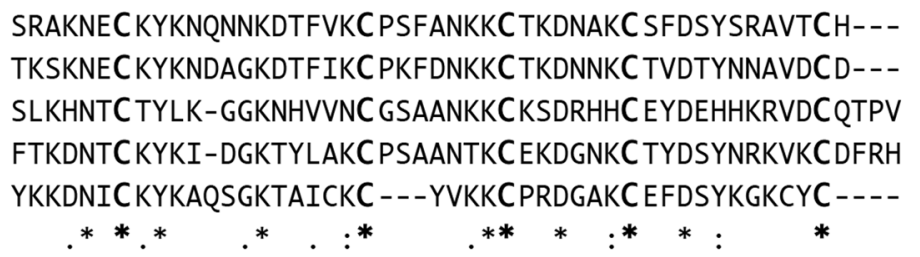

Figure 2. Amino acid alignment of Efe-AfpA from E. festucae (accession SRR493691.12929) with PAF from P. chrysogenum (accession U22944.2), PgAFP from P. chrysogenum (accession D0EXD3.1), NFAP from $N$. fischeri (accession AM983570), and AFP from A. giganteus (accession X60771). The experimentally determined $\mathrm{N}$-terminus of each protein is in bold larger font size. The conserved cysteines are also in bold larger font size. Breaks in the sequences were introduced to indicate the predicted signal peptide cleavage sites, the proposed propeptide cleavage sites, and the proposed final processing sites. An asterisk indicates identical residues in all sequences, a ":" indicates strongly conserved residues (score $>0.5$ ), and a "." indicates weaker conserved residues $(\text { score }<0.5)^{65}$.

16 to 19 amino acids downstream of the signal peptide cleavage site, indicating there is additional processing of these proteins ${ }^{21,23,26,27}$.

The most N-terminal peptide detected from Efe-AfpA was AETGILITYEGTCSR (TIC 6.3e5). However, the peptide ITYEGTCSR was considerably more abundant, with a TIC of 3.3e7, suggesting it was the N-terminus of the predominant form of the protein. The A. giganteus AFP was reported to exist in 2 forms, with the $\mathrm{N}$-terminus of the predominant form being the alanine indicated in Fig. $2^{28}$. However, on short culture times of $48-60 \mathrm{~h}$, a longer minor component with 6 additional amino acids at the $\mathrm{N}$-terminus was detected in addition to the major form. Similarly, the Efe-AfpA minor form also had an additional 6 amino acids at the N-terminus. Based on a comparison of fifteen secreted proteins from Aspergillus spp., Martinez-Ruiz et al. ${ }^{28}$ identified the common tetrapeptide LDAR at the C-terminus of the propeptides of those secreted proteins. A similar sequence is found in all the antifungal proteins shown in Fig. 2. This sequence does not conform to the canonical dibasic kexin cleavage site of $(\mathrm{K} / \mathrm{R}) \mathrm{R}$ and is not recognized by the program ProP designed to detected propeptide cleavage sites $^{29}$. However, the propeptides of some fungal secreted proteins are cleaved by yapsins, which are aspartic peptidases that can cleave C-terminal to both mono- and dibasic residues ${ }^{30}$. If the LDAR tetrapeptide is indeed the C-terminus of the E. festucae and A. giganteus antifungal protein propeptides, that would suggest that the other antifungal proteins may also be secreted as longer forms that subsequently are proteolytically cleaved to the mature form. However, such longer forms have only been identified in A. giganteus and now in E. festucae. The longer form of the A. giganteus AFP was considerably less active than the mature form ${ }^{28}$, as was the longer form of Efe-AfpA (described below).

The mature form of Efe-AfpA has 55 amino acids with a molecular weight of 6,278 Daltons, and a pI of 8.9. The difference in the calculated molecular weight of the mature Efe-AfpA of approximately $6.3 \mathrm{kDa}$ and its migration in SDS-PAGE at approximately $10 \mathrm{kDa}$ is similar to that reported for PgAFP, which was attributed to the extreme $\mathrm{pI}$ of the protein ${ }^{26}$. NMR solution structures have been determined for AFP and PAF and both proteins have compact structures held together with disulfide bonds in the hydrophobic interior core of the protein, although the precise cysteine pairing in the disulfide bonds could not be determined ${ }^{31,32}$. The cysteine pairing in PAF was determined by mass spectrometry of disulfide-bonded peptides ${ }^{33}$. The cysteine residues in PAF are conserved in Efe-AfpA (Fig. 2). Based on the conserved cysteines, as well as the overall sequence similarity, it seems likely that the tertiary structure of $E f e-A f p A$ is similar to that of PAF.

Antifungal Activity of Efe-AfpA Partially Purified from Apoplastic Proteins. Efe-AfpA was partially purified from the crude apoplastic proteins of endophyte-infected plants through hydrophobic interaction chromatography (Fig. $3 \mathrm{~A}$ ). The prominent band at about $10 \mathrm{kDa}$ in the partially purified sample was confirmed to be $E f e$-AfpA by protein sequencing. The partially purified Efe-AfpA had activity against the Sclerotinia homoeocarpa fungus in a plate assay as seen by a zone of no growth of the fungus at the site of Efe-AfpA application (Fig. $3 \mathrm{~B}$ ).

Expression of Efe-afpA in Pichia pastoris. Since the antifungal activity of Efe-AfpA isolated from apoplastic proteins was confirmed, it was expressed in the yeast Pichia pastoris in order to obtain larger quantities of protein for functional characterization. The similar antifungal proteins from P. chrysogenum (PAF), A. giganteus (AFP), and $N$. fischeri (NFAP) have been successfully expressed in P. pastoris ${ }^{32,34,35}$. Coding sequences of the long form (designated $E f e-a f p A-2$ ) and the major mature form (designated Efe-afpA-3) of Efe-AfpA were cloned into the $P$. pastoris expression vector $\mathrm{PPICZ} \alpha \mathrm{A}$, a vector designed to target the recombinant protein for secretion 

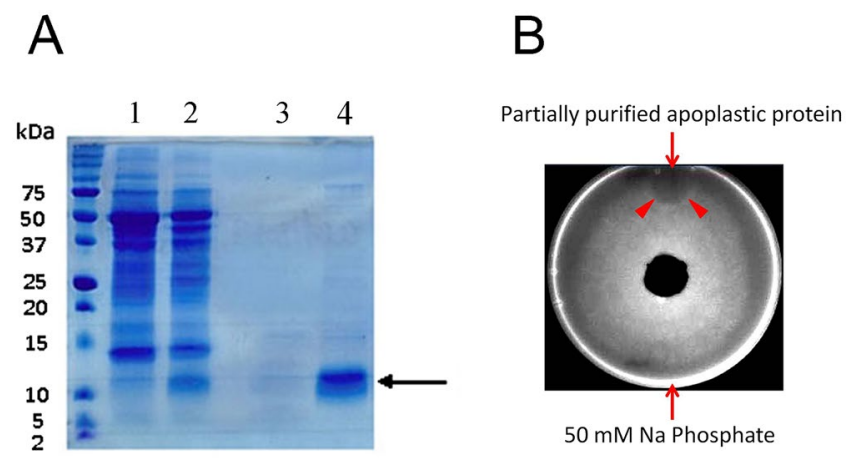

Figure 3. Partial purification of Efe-AfpA from apoplastic proteins and its activity against the dollar spot fungus. (A) SDS-PAGE analysis of partially purified Efe-AfpA from apoplastic proteins. Lane 1, total apoplastic proteins from leaves of endophyte-free plants; Lane 2, total apoplastic proteins from leaves of endophyteinfected plants; Lane 3, unbound fraction from the HiTrap phenyl HP column; Lane 4, desalted proteins eluted from the HiTrap phenyl $\mathrm{HP}$ column with $50 \mathrm{mM} \mathrm{NaPO}_{4}, 1 \mathrm{M}\left(\mathrm{NH}_{4}\right)_{2} \mathrm{SO}_{4}(\mathrm{pH}$ 7.0). Arrow indicates band cut for protein sequencing. (B) Plate assay of antifungal activity of the partially purified Efe-AfpA from apoplastic proteins against the dollar spot fungus. A plug of the dollar spot fungus was placed in the center of the plate and $30 \mu \mathrm{L}\left(1 \mu \mathrm{g} \mathrm{\mu L} \mathrm{L}^{-1}\right)$ of $E f e-A f p A$ and $50 \mathrm{mM} \mathrm{NaPO}_{4}$ were spotted at opposite ends of the plate. Arrow indicates region of growth inhibition at position of Efe-AfpA application.

\section{Culture filtrate}

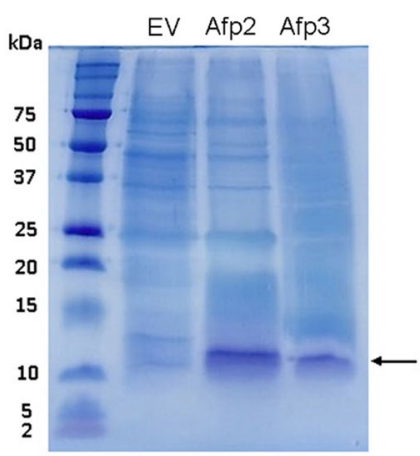

\section{Purified}

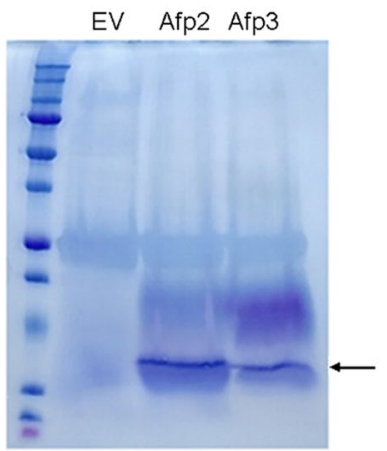

Figure 4. Expression of the Efe-afpA-2 and Efe-afpA-3 constructs in P. pastoris. SDS-PAGE of proteins in the methanol-induced $P$. pastoris culture filtrates and the partially purified Efe-AfpA-2 and Efe-AfpA-3 proteins. Arrows indicates $E f e$-AfpA proteins. EV, proteins from the empty vector control transformant; Afp2, proteins from the Efe-afpA-2 longer form construct; Afp3, proteins from the Efe-afpA-3 mature form construct.

from the yeast cells. P. pastoris does not secrete high levels of endogenous proteins, so the expressed recombinant protein is generally the major protein in the culture filtrate.

Both recombinant forms of Efe-AfpA were indeed the major proteins recovered in the culture filtrates concentrated through a $10 \mathrm{kDa}$ molecular weight cutoff centrifugal filter (Fig. 4). Efe-AfpA-2 and Efe-AfpA-3 were partially purified from the culture filtrate by first passing through a $30 \mathrm{kDa}$ molecular weight cutoff centrifugal filter and then concentrating the flow-through with a $10 \mathrm{kDa}$ molecular weight cutoff centrifugal filter (Fig. 4). Peptide sequencing of the major band at the expected size and the diffuse band at about $15 \mathrm{kDa}$ from the partially purified proteins revealed both were Efe-AfpA. A similar diffuse higher molecular weight band was seen in the purification of AFP from A. giganteus and was attributed to a dimer with rearranged disulfide bonds ${ }^{28}$. The protein band at about $25 \mathrm{kDa}$ seen in the partially purified samples from the empty vector as well as the Efe-afpA constructs was sequenced and identified as triosephosphate isomerase, whose predicted molecular weight is $27 \mathrm{kDa}$. Triosephosphate isomerase would not be expected to be secreted and is likely found in the culture filtrate due to some cell lysis, as has been reported previously for methanol-induced cultures of P.pastoris ${ }^{36}$.

Antifungal Activity of Efe-AfpA Expressed in Pichia pastoris. Efe-AfpA-2 and Efe-AfpA-3 purified from $P$. pastoris culture filtrates as described above were tested for antifungal activity against $S$. homoeocarpa in two different assays. Proteins purified in the same way from the culture filtrate of the empty vector transformant were used as controls. Since S. homoeocarpa does not produce spores, all assays were performed on S. homoeocarpa mycelium maintained on PDA. 


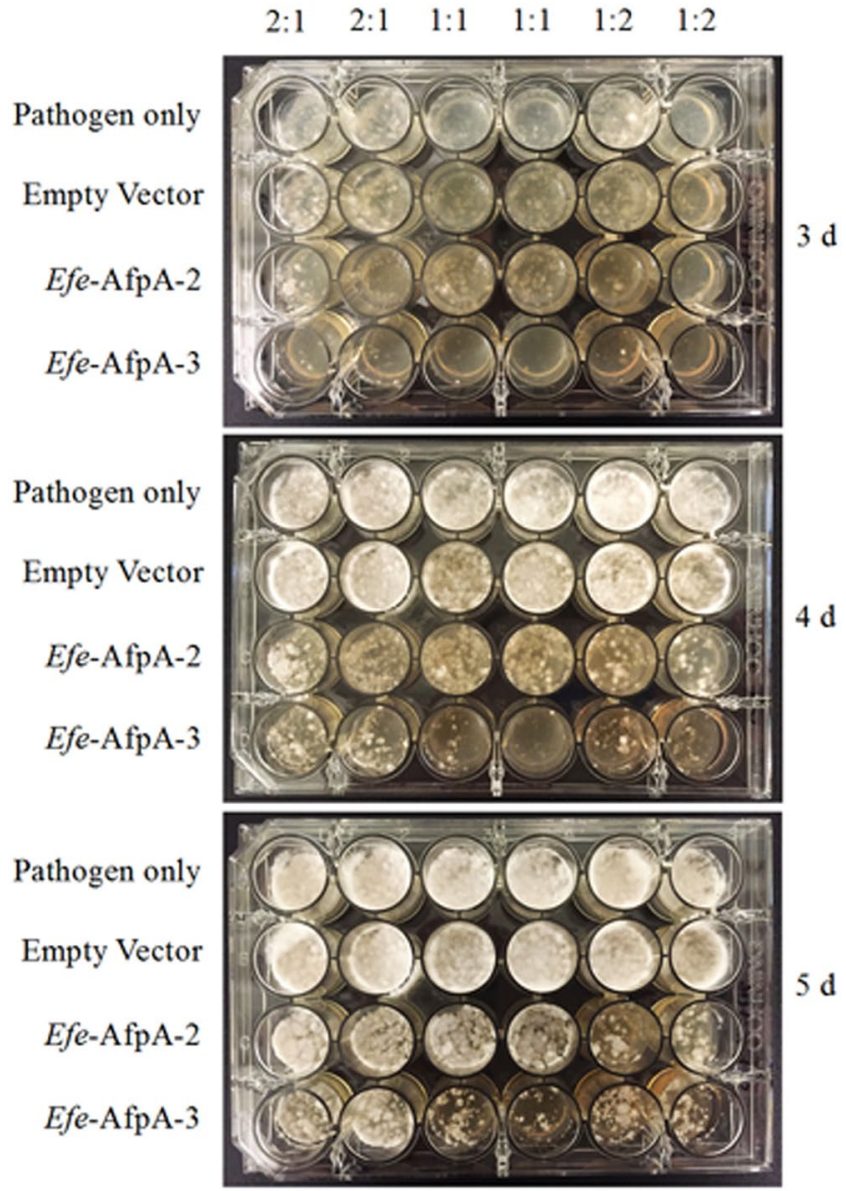

Figure 5. Antifungal activity assay of $E f e-A f p A-2$ and $E f e-A f p A-3$ purified from $P$. pastoris culture filtrates against $S$. homoeocarpa 3-5 days after inoculation. Different volumes of a $S$. homoeocarpa slurry were mixed with the purified proteins in 2:1, 1:1, and 1:2 ratios and plated onto PDA in a 24 well plate. The amounts of purified protein in the mixture are $30 \mu \mathrm{g}, 45 \mu \mathrm{g}$, and $60 \mu \mathrm{g}$, respectively.

In a qualitative assay for antifungal activity, S. homoeocarpa mycelium was gently homogenized to a slurry, mixed with $E f e-A f p A-2$ and $E f e-A f p A-3$ in different volume to volume (v:v) ratios, and the mixture applied to PDA in a 24-well plate (Fig. 5). Inhibition of growth of S. homoeocarpa by both forms of Efe-AfpA could be seen by 3 days after plating. The empty vector proteins had no effect on the growth of the pathogen. By 4 days after plating the pathogen only and empty vector samples had completely filled the wells whereas inhibition of growth was apparent for both $E f e-A f p A$ forms at all v:v ratios. Efe-AfpA-3 resulted in the greatest inhibition of the pathogen.

In a quantitative assay for antifungal activity, different concentrations of $E f e-A f p A-2$ and $E f e-A f p A-3$ were incorporated into $35 \mathrm{~mm}$ agar plates and $0.5 \mathrm{~cm}$ plugs of $S$. homoeocarpa were placed in the center of the plate. Representative plates of the triplicate samples are shown in Fig. 6. With Efe-AfpA-2 only the highest concentration, $400 \mu \mathrm{g} \mathrm{mL} \mathrm{g}^{-1}$, resulted in inhibition of growth of $S$. homoeocarpa, whereas Efe-AfpA-3 resulted in inhibition at all concentrations except $2 \mu \mathrm{g} \mathrm{mL}^{-1}$.

Efe-AfpA permeabilizes the cell membranes of S. homoeocarpa. The mechanism of the observed inhibition of growth of $S$. homoeocarpa by Efe-AfpA was investigated by using the viability stains SYTOX Green and Evans blue. SYTOX Green is a fluorescent nucleic acid stain that can only penetrate cells with compromised plasma membranes, and will not penetrate live cells. Evans blue is a non-fluorescent azo dye that also can only enter cells with damaged plasma membranes. The results of these viability assays are shown in Fig. 7. After $2 \mathrm{~h}$ of incubation with Efe-AfpA-3, the S. homoeocarpa mycelia exhibited intense fluorescence of SYTOX Green, whereas mycelia treated with the empty vector proteins or untreated mycelia only showed a low amount of fluorescence (Fig. 7A). Similarly, treatment of S. homoeocarpa with Efe-AfpA-3 resulted in uptake of Evans blue whereas treatment with the empty vector proteins had no effect (Fig. 7B).

The E. festucae Antifungal Protein Gene Is Not Present in Most Epichloë Genomes. We previously reported that genes similar to the E. festucae antifungal protein gene were not found in most Epichloë spp. for which whole genome sequences were available, being present in only E. festucae 2368 and E. inebrians as well as the E. festucae RC isolate used in our study ${ }^{17}$. Since then genome sequences of additional Epichloë spp. have been reported ${ }^{37}$ revealing that similar antifungal protein genes are present in the more recently sequenced 


\section{A}

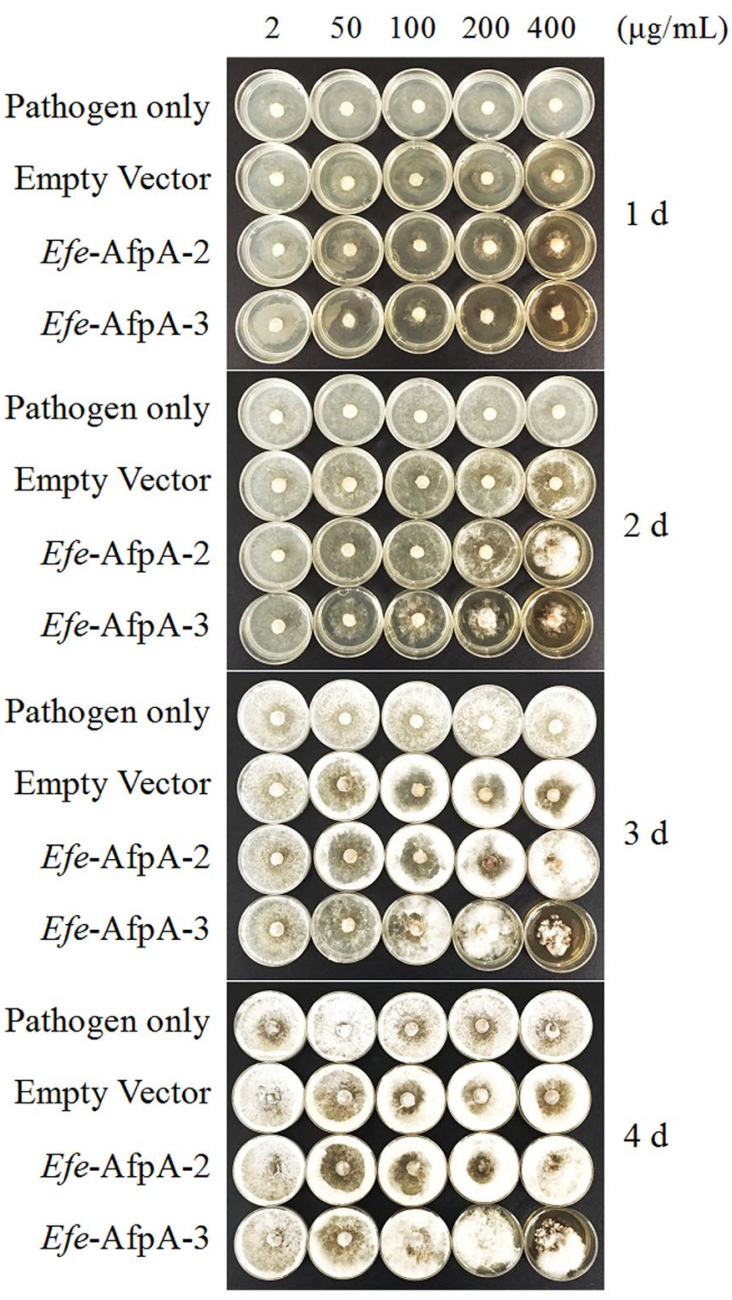

B

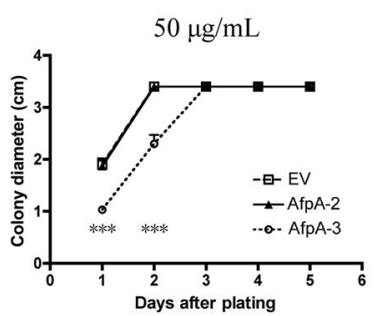

$100 \mu \mathrm{g} / \mathrm{mL}$

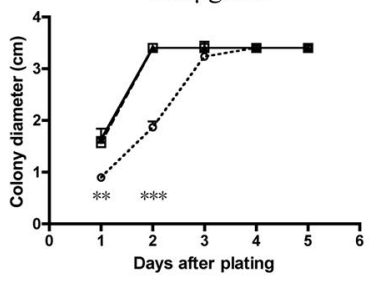

$200 \mu \mathrm{g} / \mathrm{mL}$

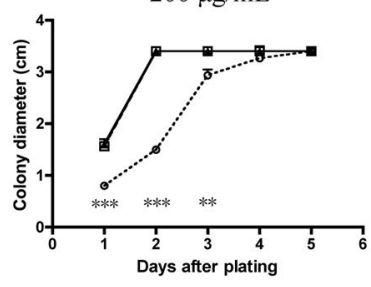

$400 \mu \mathrm{g} / \mathrm{mL}$

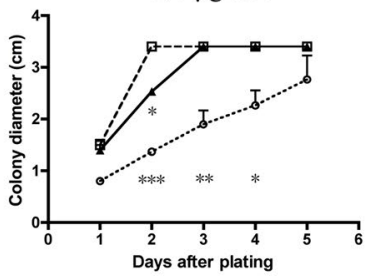

Figure 6. Antifungal activity assay of $E f e$-AfpA-2 and Efe-AfpA-3 purified from $P$. pastoris culture filtrates against $S$. homoeocarpa. The purified proteins, $2-400 \mu \mathrm{g} \mathrm{mL}^{-1}$ were incorporated into $35 \mathrm{~mm}$ PDA plates and $0.5 \mathrm{~cm}$ plugs of $S$. homoeocarpa were placed in the center of the plates. (A) Photographs of $35 \mathrm{~mm}$ plates 1-4 days after inoculation with $S$. homoeocarpa. (B) Colony diameter changes of $S$. homoeocarpa on the 35-mm plate 1-5 days after inoculation. Significant differences between constructs Efe-AfpA-2 and Efe-AfpA-3, each relative to the empty vector proteins, are indicated by $* *(P<0.001), * *(\mathrm{P}<0.01)$, and $*(P<0.05)$. Vertical bars are standard deviation values for diameter comparisons of each construct at a given day after inoculation.

genomes of E. baconii and E. aotearoae. To illustrate the evolutionary relationships among the Epichloë spp. that possess an antifungal protein gene, a maximum parsimony phylogenetic analysis was conducted of the conserved MCM7 gene, including introns, of the 17 Epichloë isolates for which whole genome sequences are available (Fig. 8). The MCM7 gene encodes a subunit of the minichromosome maintenance complex ${ }^{38}$ and is frequently used in fungal phylogenies ${ }^{39}, 40$. The E. inebrians and E. gansuensis sequences were designated as outgroups for rooting the tree since they are considered the basal Epichloë spp. ${ }^{41,42}$. The presence or absence of an antifungal protein gene in each species is indicated on the MCM7 phylogenetic tree. The patchy distribution of the presence of an antifungal protein gene throughout the Epichloë spp. suggests there have been numerous instances of gene loss.

A phylogenetic analysis of the antifungal protein gene DNA sequences, including introns, of the Epichloë spp. is shown in Fig. 9. A previous phylogenetic analysis of antifungal protein DNA coding sequences from Eurotiomycete and Sordariomycete fungi showed that the antifungal protein PAF from Pe. chrysogenum (Trichocomaceae) was more closely related to similar proteins from Sordariomycetes than those from other Eurotiomycetes ${ }^{17}$ and so is included in the phylogeny in Fig. 9. The coding sequence of the other similar antifungal protein from $\mathrm{Pe}$. chrysogenum, PgAFP, is phylogenetically close to similar sequences from other Eurotiomycetes, as would be expected ${ }^{17}$, and is not included in Fig. 9. Also included is the sequence from Pochonia chlamydosporia (Clavicipitaceae), a parasite of nematode eggs ${ }^{43}$. The sequences from the insect pathogens Beauveria bassiana and 
A
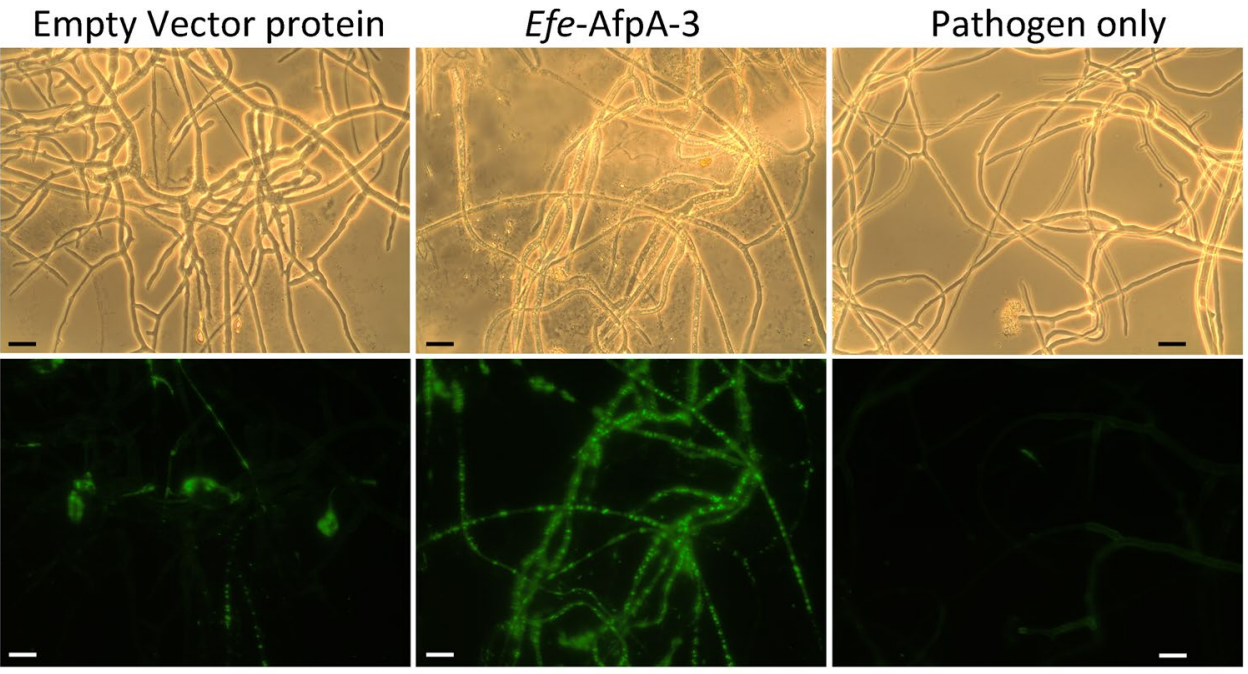

B

Empty Vector protein

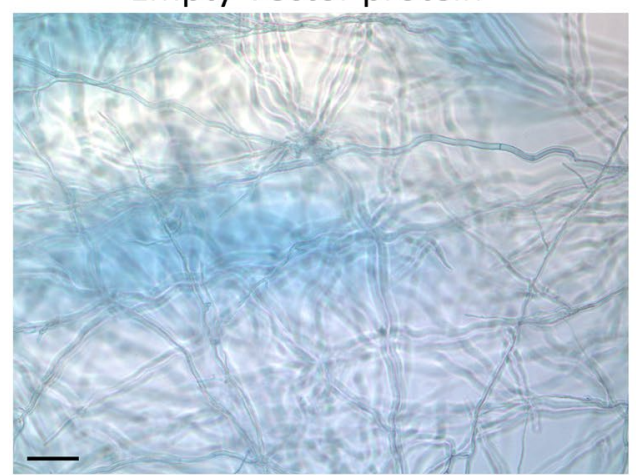

Efe-AfpA-3

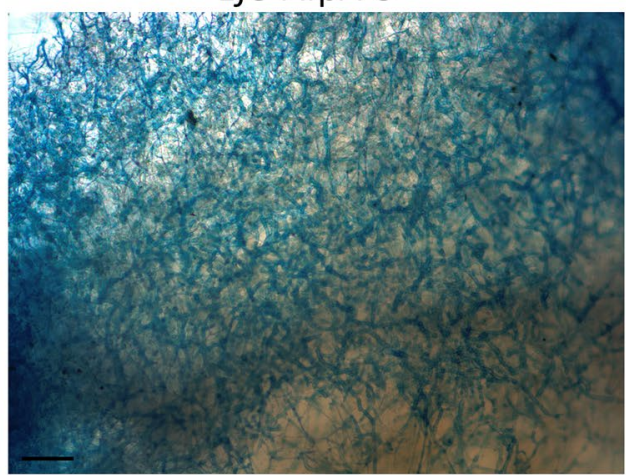

Figure 7. Microscopy of $S$. homoeocarpa mycelium treated with Efe-AfpA-3 and empty vector proteins purified from the $P$. pastoris culture filtrates. (A) Detection of SYTOX Green uptake by fluorescence microscopy. $S$. homoeocarpa was cultivated in PDB for $2 \mathrm{~d}$, and $17 \mu \mathrm{g}$ of Efe-AfpA-3 or empty vector proteins were added. After $2 \mathrm{~h}$ of incubation, fluorescence microscopy was examined in the presence of $12 \mu \mathrm{M}$ SYTOX Green. The upper panels are bright-field images and the lower panels are fluorescence images of the same fields. The scale bar is $20 \mu \mathrm{m}$. (B) Detection of Evans blue uptake by light microscopy. S. homoeocarpa in the center of a PDA coated microscope slide was treated with Efe-AfpA-3 or empty vector proteins for $1 \mathrm{~d}$ and then stained with Evans blue. The scale bar is $100 \mu \mathrm{m}$.

Cordyceps brongniartii (Cordycipitaceae ${ }^{44,45}$ were designated as outgroups for rooting the tree. In this analysis the antifungal protein gene from E. inebrians was more closely related to those of Po. chlamydosporia and Pe. chrysogenum than to the other Epichloë spp. genes. The E. inebrians sequence is $86 \%$ and $72 \%$ identical to the Po. chlamydosporia and Pe. chrysogenum sequences, respectively, whereas it is only $58-59 \%$ identical to the other Epichloë sequences (data not shown). The sequence similarity of E. inebrians to the Po. chlamydosporia and Pe. chrysogenum sequences, throughout the coding sequence as well as in the intron sequences, is readily apparent in the sequence alignment (Supplementary Fig. S2).

The phylogenetic analysis of the antifungal protein gene suggests that the evolutionary history of the gene in E. inebrians may be different from that of the other Epichloë spp. and may have involved horizontal gene transfer. Horizontal gene transfer of a large genomic island containing the PAF sequence has been reported in Penicillium spp. isolated from food environments ${ }^{46}$. In reciprocal blastn searches of the NCBI fungal genome sequences, the E. inebrians and Po. chlamydosporia antifungal protein gene sequences were the best matches to each other. There was $88 \%$ identity over a span of 913 nucleotides, including 215 bases upstream of the initiation ATG codon and 266 bases downstream of the TAG stop codon (Fig. 10). Genome sequences for 2 isolates of Po. chlamydosporia are available at NCBI, but the antifungal protein gene is present in the genome of only one of the isolates. The $E$. 


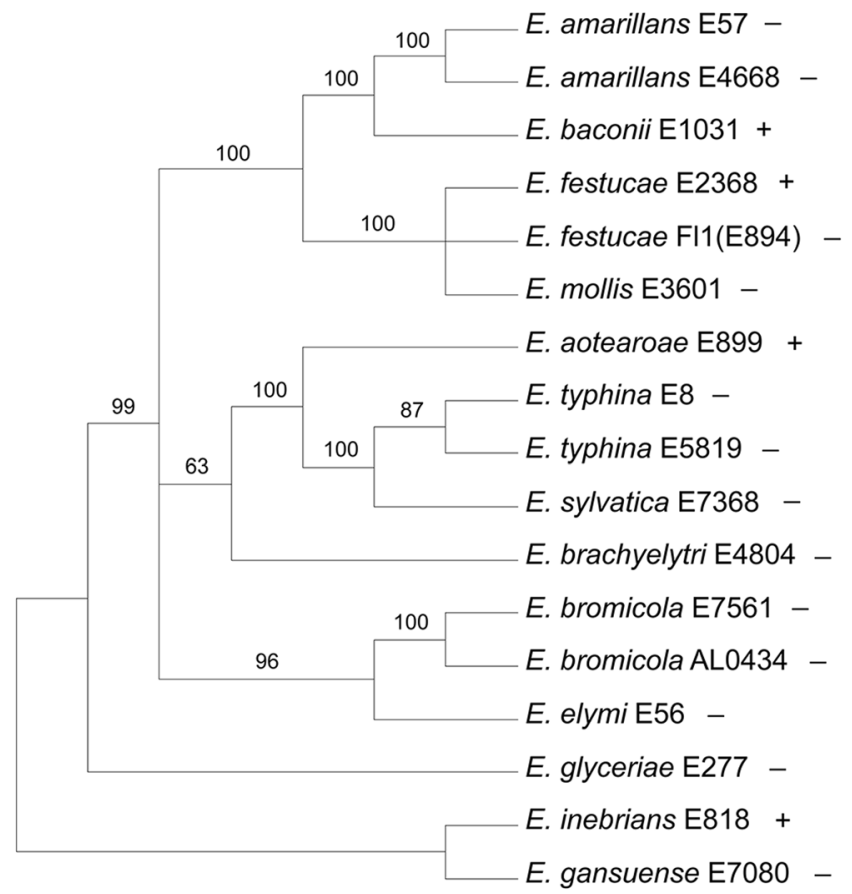

Figure 8. Rooted 50\% majority rule maximum parsimony phylogenetic tree of the MCM7 gene DNA sequences (including introns). The E. gansuensis and E. inebrians sequences were designated as the outgroups for rooting the tree. The presence $(+)$ or absence $(-)$ of an antifungal protein gene in the genome sequence is indicated for each species in the tree. The numbers at the nodes are the bootstrap percentages based on 1,000 replications. The tree was based upon 2,810 total characters, of which 2,492 were constant, 117 variable characters were parsimony uninformative, and 201 variable characters were parsimony informative. Available NCBI accession numbers of the contigs containing the sequences are: E. amarillans E57, AFRF01000144; $E$. amarillans 4668, JFGZ01000314; E. aoteraoae, JFGX01000075; E. baconii, JFGY01000038; E. brachyelytri, AFRB01001052; E. bromicola ALO4262, LBNH01000152; E. bromicola ALO434, LBNI01000418; E. elymi, AMDJ01000289; E. festucae E2368, ADFL02000041; E. festucae Fl1, AFRX02000143; E. gansuense, AFRE01000016; E. glyceriae, AFRG01000147; E. inebrians, AMDK01000026; E. sylvatica, LCTT01000222; E. typhina E8, AMDI01000042; E. typhina E5819, AFSE01000068.

inebrians and the Po. chlamydosporia antifungal protein genes appear to have a common origin that may have involved horizontal gene transfer.

Genomic Context of the E. festucae Antifungal Protein Gene. Since the phylogenetic analyses described above suggest numerous instances of antifungal protein gene loss among the Epichloë spp., as well as the likelihood of a separate evolutionary history for the gene in E. inebrians, the genomic contexts of the antifungal protein genes in the four Epichloë spp. were compared. Efe-afpA is the lone gene, flanked by repeated sequences, on a 21504 bp genome sequence contig of E. festucae 2368 (Fig. 11) $)^{5}$ (http://csbiol.csr.uky.edu/ef2011/gbrowse/ ef $/ \mathrm{q}=$ scaffold00268). The high A/T regions of the contig are the repeated sequences. Since Efe-afpA is the single gene on the contig it is not possible to determine if the gene order is syntenic with the similar genes in the other Epichloe spp. Similarly, the E. aotearoae, E. baconii, and E. inebrians antifungal protein genes are also located on small contigs of 5425,1982 , and $7529 \mathrm{bp}$, respectively.

\section{Discussion}

Here we have reported the characterization of an E. festucae antifungal protein that is highly expressed in the symbiotic association with its grass host, strong creeping red fescue. The transcript for the protein had been previously reported as being the second most abundant fungal transcript in infected strong creeping red fescue ${ }^{17}$ and, based on the peptide sequencing reported here, the protein itself is abundant in the plant apoplast. The abundance of the antifungal protein in the host plant's apoplast suggests it could come into contact with invading fungal pathogens and may therefore be a factor in the fungal disease resistance observed in E. festucae-infected strong creeping red fescue. This hypothesis is supported by the antifungal activity assays of the purified protein from the apoplastic proteins and the recombinant protein purified from the culture filtrate of the yeast $P$. pastoris. Viability assays of $S$. homoeocarpa after treatment with Efe-AfpA using SYTOX Green and Evans blue suggested that $E f e$-AfpA inhibited growth of the pathogen by causing damage to the plasma membrane. Similar results were found for AFP from A. giganteus ${ }^{47}$.

Two versions of the Efe-AfpA protein were expressed in P. pastoris, the mature form and a version with a 6 amino acid N-terminal extension that was also detected among the apoplastic proteins. Both forms did have antifungal activity, but the mature form had considerably more activity. Efe-AfpA and the similar antifungal 
E. festucae E2368

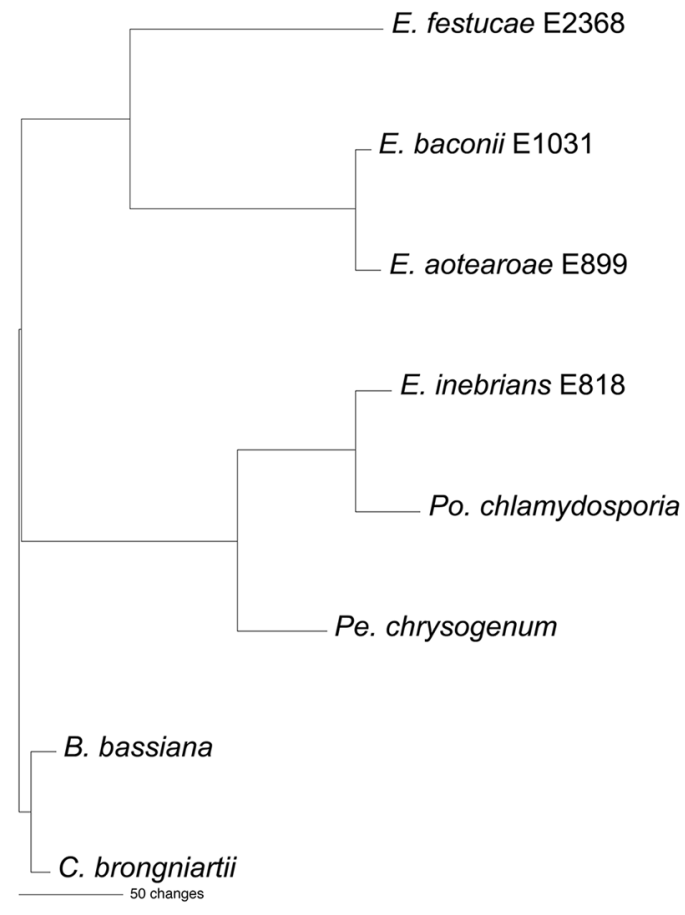

Figure 9. The single most parsimonious phylogenetic tree from an exhaustive search of antifungal protein gene DNA sequences (including introns). The B. bassiana and C. brongniartii sequences were designated as the outgroups for rooting the tree. The tree was based upon 435 total characters, of which 161 were constant, 55 variable characters were parsimony uninformative, and 219 variable characters were parsimony informative. NCBI accession numbers for the sequences are: B. bassiana ARSEF 2860, ADAH01000656; C. brongniartii RCEF 3172, AZHA01000055; E. aotearoae 1229, JFGX01000916.1; E baconii 200745, JFGY01002353.1; E. festucae E2368, ADFL02000476.1; E. inebrians E818, AMDK01000862.1; Pe. chrysogenum, U22944.2; Po. chlamydosporia 123, AOSW01001534.1.

proteins from other fungal species are all secreted proteins with a signal sequence, a pro-sequence, and apparently an additional short amino acid sequence that is processed after secretion from the cell. Many proteins have pro-sequences, presumably for proper folding ${ }^{48}$. Mature PAF, lacking the pro-sequence, expressed in bacteria had no activity suggesting a requirement for the pro-sequence for proper folding ${ }^{27}$. However, when mature forms of $E f e-A f p A$, and the similar PAF, AFP, and NFAP proteins were expressed in P. pastoris, all lacking the pro-sequence, they could fold into active forms ${ }^{32,34,35}$. Apparently the eukaryotic cellular environment of $P$. pastoris supports proper folding of the proteins even in the absence of the pro-sequences.

To confirm the involvement of the E. festucae antifungal protein in the observed disease resistance in planta, a gene knockout was attempted. The gene knockout strategy was based on the homologous recombination strategy that has been successful for other E. festucae genes ${ }^{49}$ using hygromycin resistance as a selectable marker. However, no knockouts were recovered from screening over 200 hygromycin resistant transformants. This is likely attributable to the unusual genomic context of the antifungal protein in which the small gene is surrounded by repeated sequences. Homologous recombination in filamentous fungi typically requires long regions of homol$\mathrm{ogy}^{50}$. Successful gene knockouts in E. festucae generally have 1.5 to $2.5 \mathrm{~kb}$ of flanking sequences surrounding the selectable marker and result in 1-25\% of transformants being knockouts ${ }^{49}$. For the E. festucae antifungal protein, only 598 and $634 \mathrm{bp}$ of upstream and downstream unique sequences, respectively, were available for producing the knockout construct. The short regions of homology are likely the reason no gene knockouts were obtained. In the future, another approach will be required for generating an antifungal protein gene knockout.

An E. festucae E437 isolate from the grass Festuca pulchella Schrad. (soft fescue) was reported to produce a small $(<3500 \mathrm{kDa})$, thermostable antifungal compound whose expression was controlled by a transcription factor designated $\mathrm{VibA}^{51,52}$. That antifungal compound differs from the Efe-AfpA described here, since Efe-AfpA was retained by a $3 \mathrm{kDa}$ molecular weight cut off (MWCO) centrifugal filter unit, whereas the antifungal compound from E. festucae E437 was not retained by $3.5 \mathrm{kDa} \mathrm{MWCO}$ dialysis tubing. In the future it will be interesting to determine if either of these two E. festucae isolates is capable of producing both antifungal compounds. The genome sequence of $E$. festucae $\mathrm{E} 437$ is not currently available.

Most Epichloë spp. for which whole genome sequences are available do not have a gene similar to Efe-afpA. Phylogenetic analysis suggested that the similar genes in E. baconii and E. aotearoae are evolutionarily related to Efe-afpA. However, the similar gene in E. inebrians was more evolutionarily related to genes in Po. chlamydosporia and Pe.chrysogenum, suggesting it may have originated from horizontal gene transfer, possibly from Po. chlamydosporia. Horizontal gene transfer is considered an important factor in fungal genome evolution ${ }^{53}$ and extensive horizontal gene transfer was reported between Magnaporthales and Colletotrichum ${ }^{54}$. There have been few 
E. inebrians $t \mathrm{ccataagctgcatacccaaccccgagatggcctgtatcggact} \mathrm{tgagct} \mathrm{tggccgcctcctatagagctcacaatat} c 80$ Po. chlamydosporia t c cataagctgcatacctaaccccgagatggcctgcatcggactcgggctttgcaaccacctatagaggtcacaacatta 80

E. inebrians tctatataaggggct tcacatcgctccaatctccaagcat tcat tccattaacagcactccct tctcagatactccaa 160 Po. chlamydosporia tctatat a aggaggct tcacatcgctccaatctccaag cat taat tccat-aacaacact tcct tctcagatactctaa 159

E. inebrians $\mathrm{tccatcttt} \mathrm{ctaccactttgactttcgcaagaccaccatct} \mathrm{taagctctt} \mathrm{accatgcagatcaccgcagttggccttt} 240$ Po. chlamydosporia tccatct t tctaccact t gact tccgtaagactaccatct tagctct t gccatgcagatcat- - - $\bar{a} a \overline{t g g c c t t t} 236$

E. inebrians tcctctt $\overline{c g c t g c} a \overline{a t g g g c g t g g t a g c c a c c c c} a a t \overline{t a t t} g a \overline{t c c} g a \overline{t c c a a c g g c c t c g a t g c t a g a g a t g a g g c t} 320$ Po. chlamydosporia tcct t tgcgctgcgatgggcgtggtagccacccc-..tattaa t tcccagtccaacggcctcgatgctagagatgaggct 313

E. inebrians ggcatccttatcacttactggggagtgagtaacatgaacat tccataccttgggtactcact tgggaat taccgctaac 400 Po. chlamydosporia ggcatccttatcacttactggggagtgagtaacataacat tccgtaccctaggtgctcact taggagt acagctaac 393

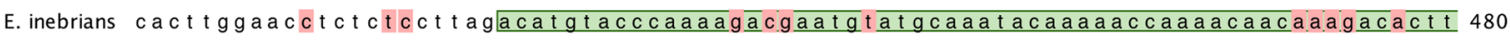
Po. chlamydosporia cact tggaact tctc- - ct t agacatgtacccaaaca aaatgaatgcaa t acaaaaccaaacaacgggaactct t 471

E. inebrians $\mathrm{ttgtcaagtgcccttt} t \mathrm{tcaataat}$ agagggtaagt tccatt taacccccaagtagccacccaggttgcacccgtgc 560 Po. chlamydosporia $\overline{\mathrm{tgtcaagtgcccttt}} \overline{\mathrm{ttc} g a t a a c a a g a a g t a a a t c t c a t t-a g t c c c t a a g t a g c c a c c t t g g t t g c a c t c g t g t} 550$

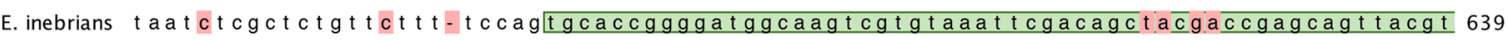

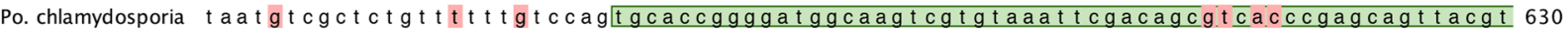

E. inebrians gttactagatggtctctacgatagctagcgtat tcaatcgtt tctagtct tcct tgtgctagtcataagcaagatgccgt 719 Po. chlamydosporia gttactagatggtctctacgatagctaccgtatctaagcgct tccagtct tccttgtgctagtaataag--- - atgccgt 706

E. inebrians gtgactctcgaagcctactacgttacatctagaagattcggaacaagaatgcttcgtgtatattgggtgtgatagacg 799 Po. chlamydosporia gtaaat c c cgaagcctgctacgtgacatctagaagaat tgggaacaaata-- - t tcgtgtatat tgggtgtaatacacg 782

E. inebrians ccaact tggtatctatgtagct cgactgttggtgaattggtt t tacgaacactctcgtgtaatatctggctagatat 879 Po. chlamydosporia ctaact tagtatctatgtagct t $\mathrm{tgactgttggtgaat}$ agt t t tacgaacactctcat t a a t t gtagctagatat 862

E. inebrians $\mathrm{t} t \mathrm{gtgct} t \mathrm{at} \mathrm{caagaaacgt} t \mathrm{tgttgactaccta} 913$

Po. chlamydosporia $t \mathrm{tg} \mathrm{tgct} t \mathrm{atcaagaaacgatcattgactaccta} 896$

Figure 10. Similarity of the E. inebrians and Po. chlamydosporia antifungal protein DNA sequences extends through the upstream and downstream regions of the gene. The antifungal protein coding sequences are boxed in green. Differences between the two sequences are boxed in red.

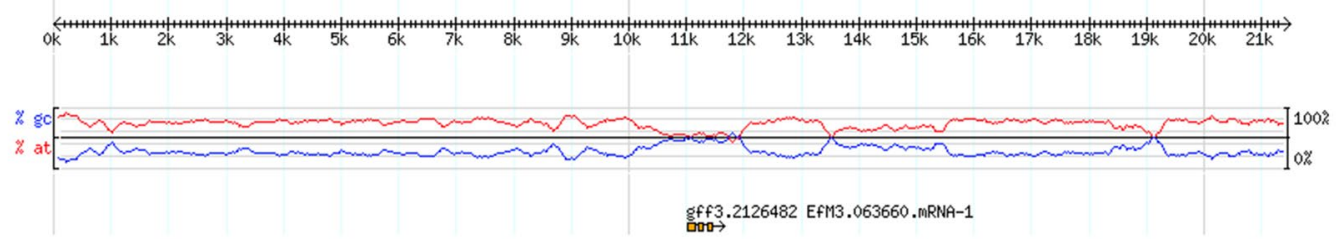

Figure 11. Efe-afpA is the only gene on a genome sequence contig consisting mainly of repeated sequences. Screenshot of E. festucae E2368 supercontig 268 from the Epichloë festucae Genome Project website ${ }^{5}$ (http:// csbio-l.csr.uky.edu/ef2011/gbrowse/ef/).

reports of horizontal gene transfer in Epichloë spp. An Epichloë toxin gene similar to the bacterial insect toxin genes makes caterpillars floppy ( $m c f 1$ and $m c f 2$ ) was proposed to have originated from horizontal gene transfer from a bacterial donor since at that time, among the hundreds of fungal genome sequences available, only Epichloe spp. had such a gene ${ }^{41}$. Since then more fungal genomes have been sequenced and $m c f$-like genes are found in a few additional fungal genomes. However, the most similar to the Epichloë spp. mcf-like gene are in the distantly related phylum Basidiomycota, suggesting that the direct donor to the Epichloë spp. may have been another fungal species, rather than a bacterial species. An extensive computational analysis of the E. festucae genome did not identify any likely horizontally transferred genes ${ }^{55}$.

In summary, the abundant secreted protein Efe-AfpA was shown to have activity against an important fungal turfgrass pathogen, S. homoeocarpa, the causal agent of dollar spot disease. Efe-AfpA may therefore be an important factor in the disease resistance observed in this symbiotic association between E. festucae and its host grass, strong creeping red fescue. By protecting its grass host from fungal pathogens the fungal endophyte E. festucae is sustaining and protecting its own living environment.

\section{Methods}

Plant and Fungal Materials. The strong creeping red fescue (Festuca rubra subsp. rubra) plants used in this study, S1139E- and S1139 Rose City (S1139RC), have been described previously ${ }^{56}$. The endophyte-infected plant S1139RC was generated by inoculating an isolated tiller of the uninfected strong creeping red fescue plant S1139E- with the Rose City isolate of E. festucae, which was isolated from an unrelated endophyte-infected strong creeping red fescue. The plants S1139E- and S1139RC are thus the same plant genotype. Plants were clonally propagated and maintained in the greenhouse. These plants have been stably maintained in the greenhouse for 


\begin{tabular}{|l|l|}
\hline Forward primer, $\mathbf{5}^{\prime}-\mathbf{3}^{\prime}$ & Reverse primer, $\mathbf{5}^{\prime} \mathbf{-} \mathbf{3}^{\prime}$ \\
\hline Cloning into $\mathrm{pPICZ} \alpha \mathrm{A}$ & AAGCTGGCGGGCCGCCTAATGACACGTGACAGCTC \\
\hline Efe-afpA-2 & GTATCTCTCGAGAAAAGAGCGGAGACTGGCATTCTGA \\
\hline Efe-afpA-3 & AAGCTGGCGGGCCGCCTAATGACACGTGACAGCTC \\
\hline GTATCTCTCGAGAAAAGAATCACGTATGAAGGAACATGTT & Deletion of propeptide and Kex2 sites of the $\alpha$-factor signal sequence \\
\hline Efe-afpA-3 & AGCTAATGCGGAGGATGC \\
\hline ATCACGTATGAAGGAACATGTTC & \\
\hline Sequencing recombinant plasmids & \\
\hline $5^{\prime}$ AOX1 primer & \\
\hline GACTGGTTCCAATTGACAAGC & \\
\hline
\end{tabular}

Table 1. Sequences of oligonucleotide primers used in this study.

over 15 years. The endophyte status of the plants was confirmed microscopically ${ }^{57}$ before initiation of this study. The E. festucae Rose City isolate (E. festucae RC) was isolated from the endophyte-infected S1139RC plant by plating surface-sterilized leaf sheath tissue on potato dextrose agar (Difco Laboratories, Detroit, MI, USA).

Isolation of Apoplastic Proteins. Isolation of apoplastic proteins was conducted by using a modification of the method previously described by Moy et al. ${ }^{58}$. Two g of leaves and leaf sheaths were cut into 2 -cm segments, rinsed, and vacuum infiltrated for $30 \mathrm{~min}$ with $20 \mathrm{~mL}$ of $100 \mathrm{mM}$ Tris- $\mathrm{HCl}, \mathrm{pH} 8.0,50 \mathrm{mM}$ dithiothreitol, $10 \mathrm{mM}$ ascorbic acid, and $1 \mathrm{mM}$ Pefabloc SC protease inhibitor (Sigma-Aldrich, St. Louis, MO, USA). The tissue was washed with water three times, blotted dry, and collected in a 10-cc syringe. The syringe was placed in a $30 \mathrm{~mL}$ Corex glass tube and centrifuged at 2,000 $\times \mathrm{g}$ for $10 \mathrm{~min}$ at $4^{\circ} \mathrm{C}$. The apoplastic fluid was collected in a microfuge tube. Protein concentrations were determined by using the Bio-Rad Protein Assay Dye Reagent Concentrate (Bio-Rad Laboratories, Hercules, CA, USA).

For sodium dodecyl sulfate-polyacrylamide gel electrophoresis (SDS-PAGE) analysis, protein samples were mixed with $5 x$ SDS sample buffer $(5: 1, \mathrm{v} / \mathrm{v})^{59}$, then boiled for $5 \mathrm{~min}$ and subjected to electrophoresis in $16 \%$ polyacrylamide gels. Gels were stained with Coomassie Blue to visualize protein bands. Gels were destained, and then dried using a modified protocol from Moghaddam and Reinton ${ }^{60}$ as described previously ${ }^{61}$. Gels were soaked in a solution of $50 \%(\mathrm{v} / \mathrm{v})$ methanol and $20 \%(\mathrm{v} / \mathrm{v})$ polyethylene glycol 400 (PEG-400). Cellophane sheets, first soaked in water, were used to sandwich the gels in a gel-drying frame. Gels were dried at room temperature overnight.

Peptide Sequencing. For peptide sequencing, the target band was excised from the SDS-PAGE gel and washed twice with $50 \%$ acetonitrile in water. Sequence analysis of peptides in the band from the endophyte-infected apoplastic proteins was performed at the Harvard Mass Spectrometry and Proteomics Resource Laboratory (Cambridge, MA, USA) by microcapillary reverse-phase HPLC nano-electrospray tandem mass spectrometry (LC/MS/MS) on a Thermo LTQ-Orbitrap mass spectrometer. Peptide sequence analysis of the antifungal protein partially purified from the apoplastic proteins was performed at the Biological Mass Spectrometry Facility at Rutgers University (Piscataway, NJ, USA) by LC/MS/MS on a Thermo Q Exactive HF mass spectrometer with a Dionex U-3000 rapid separation nano LC system.

Partial Purification of Efe-AfpA From the Apoplastic Proteins. Apoplastic fluid from $4 \mathrm{~g}$ of leaves was collected as described above and mixed with an equal volume of $100 \mathrm{mM} \mathrm{NaPO}_{4}, 3.0 \mathrm{M}\left(\mathrm{NH}_{4}\right)_{2} \mathrm{SO}_{4}(\mathrm{pH} 7.0)$. The sample was then applied to a 1-mL HiTrap phenyl HP column (GE Healthcare Life Sciences, Piscataway, NJ) and the column washed with $5 \mathrm{~mL}$ of $50 \mathrm{mM} \mathrm{NaPO}_{4}, 1.5 \mathrm{M}\left(\mathrm{NH}_{4}\right)_{2} \mathrm{SO}_{4}(\mathrm{pH} 7.0)$ to remove unbound proteins. Efe-AfpA was eluted with five $\mathrm{mL}$ of $50 \mathrm{mM} \mathrm{NaPO}_{4}, 1 \mathrm{M}\left(\mathrm{NH}_{4}\right)_{2} \mathrm{SO}_{4}(\mathrm{pH} 7.0)$. One $\mathrm{mL}$ aliquot fractions were collected. The buffer was exchanged to $50 \mathrm{mM} \mathrm{NaPO}_{4}(\mathrm{pH} 7.0)$ and the protein was concentrated by using Amicon Ultra-0.5 mL $3 \mathrm{kDa}$ centrifugal filters (EMD Millipore, Billerica, MA, USA).

Production of the E. festucae Antifungal Protein in Pichia pastoris. Efe-afpA was expressed in Pichia pastoris by using the EasySelect Pichia Expression kit (Invitrogen by Life Technologies, Carlsbad, CA, USA). Two expression vectors were constructed based on the longer form of Efe-AfpA recovered from the apoplastic proteins beginning with AET, designated $E f e$-AfpA-2, and the most abundant mature form of Efe-AfpA from the apoplastic proteins beginning with ITY, designated Efe-AfpA-3. The Efe-afpA coding sequences were cloned into the Xho 1 and Not 1 sites of expression vector PPICZ $\alpha$ A, a vector designed to target the recombinant protein for secretion from the yeast cells by cloning downstream of the Saccharomyces cerevisiae $\alpha$-factor mating signal sequence. The Efe-afpA coding sequences were amplified by PCR with oligonucleotides that introduced a Xho 1 site and the Kex 2 site at the $5^{\prime}$ ends and a Not 1 site at the $3^{\prime}$ end. The Ste 13 cleavage sites were not included since the STE13 protease has been reported to inefficiently cleave the resulting protein ${ }^{34,62}$. The oligonucleotides used for PCR amplification of the coding sequences are presented in Table 1.

For both constructs, the amplification reactions were carried out in a GeneAmp 9700 thermo-cycler (Applied Biosystems, Foster City, CA, USA). The $100 \mu \mathrm{L}$ reactions contained 2X Phusion High-Fidelity PCR Master Mix with HF Buffer (Thermo Fisher Scientific, Waltham, MA, USA), 40 pmol of each oligonucleotide, and 80 pg plasmid DNA as template. The PCR reaction conditions were: an initial denaturation step at $94^{\circ} \mathrm{C}$ for $30 \mathrm{~s}$, followed 
by 30 cycles of $10 \mathrm{~s}$ denaturation at $98^{\circ} \mathrm{C}, 30 \mathrm{~s}$ annealing at $59^{\circ} \mathrm{C}$, and $30 \mathrm{~s}$ extension at $72^{\circ} \mathrm{C}$. An additional final 5 min extension at $72^{\circ} \mathrm{C}$ was performed. The PCR products were purified by using $1.8 \mathrm{X}$ Agencourt AMPure XP (Beckman Coulter, Brea, CA, USA).

The purified $E f e-a f p A$ PCR products were digested with FastDigest Xho 1 and Not 1 restriction enzymes (Thermo Fisher Scientific). The PCR products were first digested with $1 \mu \mathrm{L}$ Not 1 at $37^{\circ} \mathrm{C}$ for $30 \mathrm{~min}$, then 0.5 $\mu \mathrm{L}$ of Xho 1 was added to the reaction and the incubation continued for $4 \mathrm{~min}$. The samples were then heated at $80^{\circ} \mathrm{C}$ for $5 \mathrm{~min}$ to inactivate the enzymes. Since there is a Xho 1 site within the Efe-afpA coding sequence, the Xho 1 digest was short to facilitate recovery of partially digested PCR products. The expression vector pPICZ $\alpha$ A was also digested with FastDigest Xho 1 and Not 1 restriction enzymes (Thermo Fisher Scientific) followed by treatment with shrimp alkaline phosphatase (Affymetrix, Santa Clara, CA, USA) to prevent vector relegation. The digested PCR products and vector were purified by using 1.8 X Agencourt AMPure XP. Ligation of the digested PCR products (22 ng) and pPICZ $\alpha$ A (200 ng) was with T4 DNA ligase (Invitrogen, Carlsbad, CA, USA) overnight at room temperature.

Two $\mu \mathrm{L}$ of the ligation products were used to transform $20 \mu \mathrm{L}$ E. coli electroporation-competent TOP $10 \mathrm{~F}^{\prime}$ cells. The transformed cells were incubated in low-salt LB medium for 1 hour at $37^{\circ} \mathrm{C}$ with shaking, followed by overnight growth of cells on low salt LB medium supplemented with $25 \mu \mathrm{g} \mathrm{mL} \mathrm{mL}^{-1}$ zeocin. Transformed bacterial colonies were screened for recombinant plasmids containing the Efe-afpA coding sequence inserts by using PCR as described above. The PCR products were visualized on a $2 \% \mathrm{TBE}$ agarose gel. Plasmids from selected positive bacterial colonies were isolated and sequenced (Genewiz, Inc., South Plainfield, NJ, USA) using the 5' AOX1 sequencing primer (Table 1$)$.

Protein sequencing (described above) of the recombinant Efe-AfpA-3 revealed that the protein was not efficiently processed at the Kex 2 site since a prominent peptide recovered was RITYEGTCSR. Therefore, another construct for $E f e-a f p A-3$ was generated by deleting the $\alpha$-factor propeptide sequence including the Kex 2 site in the plasmid, such that the primary $\alpha$-factor signal sequence cleavage site was adjacent to the start of the mature Efe-afpA-3 sequence. The Q5 Site-Directed Mutagenesis Kit (New England BioLabs Inc., Ipswich, MA, USA) was used to modify the plasmid containing the mature form of Efe-afpA-3 by deleting the plasmid propeptide $\alpha$-factor coding sequence of 66 amino acids, including the Kex 2 signal cleavage site, downstream of the experimentally determined 19 amino acid $\alpha$-factor signal sequence ${ }^{63}$ (Table 1 ).

The pPICZ $\alpha$ A empty vector and the recombinant plasmids containing the Efe-afpA coding sequences were linearized by FastDigest Mss 1 (Thermo Fisher Scientific) and then purified by using 1.8X Agencourt AMPure XP. The purified linearized plasmids were transformed into P. pastoris KM71H (arg4 aox 1::ARG4) cells by electroporation. The P. pastoris cells were prepared for electroporation as described in the EasySelect Pichia Expression Kit manual. Selected transformant $P$. pastoris clones were grown and protein expression induced by methanol addition for 5 days as described in the kit manual. The culture filtrate was then collected by centrifuging at $3000 \times \mathrm{g}$ at $25^{\circ} \mathrm{C}$ for $5 \mathrm{~min}$.

Efe-AfpA-2 and Efe-AfpA-3 were partially purified from the P. pastoris culture filtrate by first removing proteins larger than $30 \mathrm{kDa}$ by passing through Amicon Ultra- 15 molecular weight cutoff $30 \mathrm{kDa}$ centrifugal filters (Sigma-Aldrich) at $3000 \times \mathrm{g}$ at $4^{\circ} \mathrm{C}$. Proteins in the flow-through were then concentrated by using Amicon Ultra15 molecular weight cutoff $10 \mathrm{kDa}$ centrifugal filters at $3000 \times \mathrm{g}$ at $4^{\circ} \mathrm{C}$. Proteins from the culture filtrate of the pPICZ $\alpha$ A empty vector control P. pastoris transformant were prepared in the same way.

Antifungal Activity Assays. S. homoeocarpa was maintained on potato dextrose agar (PDA) containing $50 \mu \mathrm{g} \mathrm{mL}^{-1}$ kanamycin. Since $S$. homoeocarpa does not produce spores, all antifungal activity assays were carried out on mycelium harvested from the PDA plates. A plate assay was used to qualitatively determine the antifungal activity of Efe-AfpA purified from apoplastic fluid against S. homoeocarpa. A thin layer of S. homoeocarpa fungus $\left(1 \mathrm{~cm}^{2}\right)$ was cut and placed at the center of a PDA plus kanamycin plate. The plate was incubated at room temperature for one day. Thirty microliters of the partially purified antifungal protein $\left(1 \mu \mathrm{g}_{\mu} \mathrm{L}^{-1}\right)$ and $30 \mu \mathrm{L}$ of $50 \mathrm{mM} \mathrm{NaPO}_{4}$ were added to opposite sides of the plate. The plate was incubated at room temperature. A zone of no growth of the pathogen, indicative of antifungal activity, was apparent after $1 \mathrm{~d}$.

A 24-well plate assay was used to qualitatively determine the antifungal activity of Efe-AfpA expressed in $P$. pastoris on $S$. homoeocarpa. Prior to use in the antifungal assays, the partially purified proteins from the $P$. pastoris culture filtrates were filter sterilized by passing through a $0.2 \mu \mathrm{m}$ syringe filter (Corning Incorporated, Corning, NY, USA). A mycelial plug of fungus $(1.5 \mathrm{~cm} \times 1.5 \mathrm{~cm})$ was cut from a $7 \mathrm{~d}$ culture of the fungus on a PDA plate and ground in $3 \mathrm{~mL}$ potato dextrose broth (PDB) by using a sterile glass dounce homogenizer. The pathogen suspension was mixed with Efe-AfpA-2, Efe-AfpA-3, or the empty vector transformant proteins purified from the $P$. pastoris culture filtrate in $2: 1,1: 1$, and 1:2 volume to volume (v:v) ratios, in a total of $60 \mu \mathrm{L}$. The amount of purified proteins in the mixtures was $30 \mu \mathrm{g}, 45 \mu \mathrm{g}$, and $60 \mu \mathrm{g}$, respectively. The pathogen/protein mixtures were spread onto PDA in a 24-well plate and incubated at room temperature.

A 35-mm petri dish plate assay was used to quantitatively determine the antifungal activity of Efe-Afp-2, Efe-Afp-3, or the empty vector transformant proteins against $S$. homoeocarpa. Three replicates each of Efe-AfpA-2, Efe-AfpA-3, or empty vector transformant proteins purified from the $P$. pastoris culture filtrate were mixed with $\mathrm{PDA}$ in final concentrations of 2, 50,100, 200, and $400 \mu \mathrm{g} \mathrm{mL}^{-1}$. Mycelial plugs of $S$. homoeocarpa $(0.5 \mathrm{~cm}$ diameter) were cut from a $7 \mathrm{~d}$ culture of the fungus on a PDA plate and placed at the center of the $35-\mathrm{mm}$ petri dishes containing the PDA-antifungal protein mixtures and incubated at room temperature. The colony diameters $(\mathrm{cm})$ were measured daily, and mean values were used to quantitatively determine inhibitory activity.

Microscopy. The SYTOX Green uptake assay was modified from a method described previously ${ }^{47}$. A mycelial plug of $S$. homoeocarpa $(0.2 \mathrm{~cm} \times 0.2 \mathrm{~cm})$ was cut from a $7 \mathrm{~d}$ culture of the fungus on a PDA plate and ground in $3 \mathrm{~mL}$ potato dextrose broth (PDB) by using a sterile glass dounce homogenizer. Two hundred $\mu \mathrm{L}$ of the fungal 
suspension were cultivated in a 96-well cell culture plate at room temperature for $2 \mathrm{~d}$. Seventeen $\mu \mathrm{g}$ of $E f e$-AfpA-3 or empty vector transformant proteins purified from the $P$. pastoris culture filtrate were added and incubated for $2 \mathrm{~h}$. SYTOX Green was added to a final concentration of $12 \mu \mathrm{M}$. Fluorescence was examined and photographed with a Carl Zeiss Axioskop microscope with the filter set at an excitation wavelength of 450 to $490 \mathrm{~nm}$ and an emission wavelength at 515 to $565 \mathrm{~nm}$.

The Evans blue staining assay was modified from Shehata et al. ${ }^{64}$. Five hundred $\mu \mathrm{L}$ of PDA plus $50 \mu \mathrm{g} \mathrm{mL}{ }^{-1}$ kanamycin was spread on a sterilized glass slide in a petri dish and allowed to solidify. A fragment of S. homoeocarpa from a $7 \mathrm{~d}$ culture of the fungus on a PDA plate was applied to the center on the agar. Thirty-five micrograms of Efe-AfpA-3 or empty vector transformant proteins purified from the $P$. pastoris culture filtrate was added to the side of the $S$. homoeocarpa fungal fragment on each slide. Slides were incubated at room temperature for $1 \mathrm{~d}$. They were then stained with a solution of $1 \%$ Evans blue for $15 \mathrm{~min}$, followed by washing with water for $15 \mathrm{~min}$, and examined and photographed with a Carl Zeiss Axioskop microscope.

Phylogenetic Analysis. The MCM7 (gene model EfM3.060700) and antifungal protein (gene model EfM3.063660) gene sequences were obtained from the Genome Project at the University of Kentucky website ${ }^{5,31}$ (http://www.endophyte.uky.edu/). The Clustal-X program was used to align the sequences ${ }^{65}$. The alignments generated by Clustal-X were modified manually to minimize gaps. The phylogenetic analysis was performed with the PAUP* program, version 4.0b10 for Macintosh. The MCM7 phylogenetic analysis was done by using the maximum parsimony full heuristic search option set to random sequence addition, tree-bisection-reconnection (TBR) branch swapping, and Multrees on, with 1000 bootstrap replications. Gaps were treated as missing data. The antifungal protein gene and protein phylogenetic trees were done by using an exhaustive maximum parsimony analysis, which returned a single most parsimonious tree in both cases.

Data Availability. All data generated or analysed during this study are included in this published article (and its Supplementary information files).

\section{References}

1. Schardl, C. L., Scott, B., Florea, S. \& Zhang, D. Epichloë endophytes: clavicipitaceous symbionts of grasses. In Plant Relationships, $2^{\text {nd }}$ Edition, The Mycota V. (ed. Deising, H.) 275-306 (Springer-Verlag, Berlin, 2009).

2. Tadych, M., Bergen, M. S. \& White, J. F. Jr. Epichloë spp. associated with grasses: new insights on life cycles, dissemination and evolution. Mycologia 106, 181-201 (2014).

3. Voisey, C. R. Intercalary growth in hyphae of filamentous fungi. Fungal Biol. Rev. 24, 123-131 (2010).

4. Kuldau, G. \& Bacon, C. Clavicipitaceous endophytes: their ability to enhance resistance of grasses to multiple stresses. Biol. Control 46, 57-71 (2008).

5. Schardl, C. L. et al. Plant-symbiotic fungi as chemical engineers: multi-genome analysis of the Clavicipitaceae reveals dynamics of alkaloid loci. PLoS Genet. 9(2), e1003323 (2013).

6. Bonos, S. A., Wilson, M. M., Meyer, W. A. \& Funk, C. R. Suppression of red thread in fine fescues through endophyte-mediated resistance. App. Turfgrass Sci. 10, 1094 (2005).

7. Clarke, B. B. et al. Endophyte-mediated suppression of dollar spot disease in fine fescues. Plant Dis. 90, 994-998 (2006).

8. Malinowski, D. P. \& Belesky, D. P. Adaptations of endophyte-infected cool-season grasses to environmental stresses: mechanisms of drought and mineral stress tolerance. Crop Sci 40, 923-940 (2000).

9. Nagabhyru, P., Dinkins, R. D., Wood, C. L., Bacon, C. W. \& Schardl, C. L. Tall fescue endophyte effects on tolerance to water-deficit stress. BMC Plant Biol 13, 127 (2013).

10. Ruemmele, B. A., Wipff, J. K., Brilman, L. \& Hignight, K. W. Fine-leaved Festuca species. In Turfgrass biology, genetics, and breeding (eds Cassler, M. D. \& Duncan, R. R.) 129-174 (Wiley, Hoboken, New Jersey, 2003).

11. Berestetski, A., Ehrig, F. \& Kastirr, U. Preliminary evaluation of turfgrass (perennial ryegrass and red fescue) cultivars for resistance to red thread disease using artificial infection. Plant Breeding 121, 493-500 (2002)

12. Tate, T. M. et al. Performance of fine fescue cultivars and selections in New Jersey turf trials. 2015 Rutgers Turfgrass Proceedings: 2015 Research Report 47, 41-58 (2016).

13. Rodriguez, R. J., White, J. F. Jr., Arnold, A. E. \& Redman, R. S. Fungal endophytes: diversity and functional roles. New Phytol 182, 314-330 (2009).

14. Burpee, L. L. \& Bouton, J. H. Effect of eradication of the endophyte Acremonium coenophialum on epidemics of Rhizoctonia blight in tall fescue. Plant Dis. 77, 157-159 (1993).

15. Welty, R. E., Barker, R. E. \& Azevedo, M. D. Reaction of tall fescue infected and noninfected by Acremonium coenophialum to Puccinia graminis subsp. graminicola. Plant Dis. 75, 883-886 (1991).

16. Hume, D. E. et al. Epichloë fungal endophytes for grassland ecosystems. In Sustainable agriculture reviews 19 (ed. E. Lichtfouse) 233-305 (Springer International Publishing, Switzerland, 2016).

17. Ambrose, K. V. \& Belanger, F. C. SOLiD-SAGE of endophyte-infected red fescue reveals numerous effects on host transcriptome and an abundance of highly expressed fungal secreted proteins. PLoS ONE 7(12), e53214 (2012).

18. Marx, F. S. basic antifungal proteins secreted from filamentous ascomycetes: a comparative study regarding expression, structure, function and potential application. App. Microbiol. Biotechnol 65, 1330142 (2004).

19. Schardl, C. L. \& Scott, B. Recommendations for gene nomenclature for Epichloë species and related Clavicipitaceae. In Epichloae, endophytes of cool season grasses: implications, utilization and biology (eds Young, C. A., Aiken, G. E., McCulley, R. L., Strickland, J. R. \& Schardl, C. L.) 84-87 (The Samuel Roberts Noble Foundation, Ardmore, Oklahoma, 2012).

20. Emanuelsson, O., Brunak, S., von Heijne, G. \& Nielsen, H. Locating proteins in the cell using TargetP, SignalP, and related tools. Nat. Protoc. 2, 953-971 (2007).

21. Whendt, S., Ulbrich, N. \& Stahl, U. Molecular cloning, sequence analysis and expression of the gene encoding an antifungal-protein from Aspergillus giganteus. Curr. Genet. 25, 519-523 (1994).

22. Meyer, V. A small protein that fights fungi: AFP as a new promising antifungal agent of biotechnological value. Appl. Microbiol. Biotechnol. 78, 17-28 (2008).

23. Kovacs, L. et al. Isolation and characterization of Neosartorya fischeri antifungal protein (NFAP). Peptides 32, 1724-1731 (2011).

24. Marx, F. et al. Cloning, structural organization and regulation of expression of the Penicillium chrysogenum paf gene encoding an abundantly secreted protein with antifungal activity. Gene 167, 167-171 (1995).

25. Hegedus, N. et al. The small molecular mass antifungal protein of Penicillium chrysogenum - a mechanism of action oriented review. J. Basic Microbiol. 51, 561-571 (2011).

26. Rodriguez-Martin, A. et al. Characterization of the novel antifungal protein PgAFP and the encoding gene of Penicillium chrysogenum. Peptides 31, 541-547 (2010). 
27. Marx, F. et al. Proper folding of the antifungal protein PAF is required for optimal activity. Res. Microbiol. 156, 35-46 (2005).

28. Martinez-Ruiz, A. et al. Characterization of a natural larger form of the antifungal protein (AFP) from Aspergillus giganteus. Biochim. Biophys. Acta 1340, 81-87 (1997).

29. Duckert, P., Brunak, S. \& Blom, N. Prediction of proprotein convertase cleavage sites. Protein Eng. Des. Sel. 17, 107-112 (2004).

30. Gagnon-Arsenault, I., Tremblay, J. \& Bourbonnais, Y. Fungal yapsins and cell wall: a unique family of aspartic peptidases for a distinctive cellular function. FEMS Yeast Res 6, 966-978 (2006).

31. Campos-Olivas, R. et al. NMR solution structure of the antifungal protein from Aspergillus giganteus: evidence for cysteine pairing isomerism. Biochemistry 34, 3009-3021 (1995).

32. Batta, G. et al. Functional aspects of the solution structure and dynamics of PAF - a highly-stable antifungal protein from Penicillium chrysogenum. FEBS J 276, 2875-2890 (2009).

33. Varadi, G. et al. Synthesis of PAF, an antifungal protein from P. chrysogenum, by native chemical ligation: native disulfide pattern and fold obtained upon oxidative refolding. Chemistry 19, 12684-12692 (2013).

34. Lopez-Garcia, B. et al. Production of the biotechnologically relevant AFP from Aspergillus giganteus in the yeast Pichia pastoris. Protein Expr. Purif. 70, 206-210 (2010).

35. Viragh, M. et al. Production of a defensin-like antifungal protein NFAP from Neosartorya fischeri in Pichia pastoris and its antifungal activity against filamentous fungal isolates from human infections. Protein Expr. Purif. 94, 79-84 (2014).

36. Huang, C.-J. et al. A proteomic analysis of the Pichia pastoris secretome in methanol-induced cultures. Appl. Microbiol. Biotechnol. 90, 235-247 (2011).

37. Schardl, C. L. et al. Genomes of plant-associated Clavicipitaceae. Adv. Bot. Res 70, 291-327 (2014).

38. Alabert, C. \& Groth, A. Chromatin replication and epigenome maintenance. Nat. Rev. Mol. Cell. Bio 13, 153-167 (2012).

39. Aguileta, G. et al. Assessing the performance of single-copy genes for recovering robust phylogenies. Syst. Biol 57, 613-627 (2008).

40. Raja, H. A., Schoch, C. L., Hustad, V. P., Shearer, C. A. \& Miller, A. N. Testing the phylogenetic utility of MCM7 in the Ascomycota. MycoKeys 1, 63-94 (2011).

41. Ambrose, K. V., Koppenhofer, A. M. \& Belanger, F. C. Horizontal gene transfer of a bacterial insect toxin gene into the Epichloë fungal symbionts of grasses. Sci. Rep. 4, 5562 (2014).

42. Chen, L. et al. Two distinct Epichloë species symbiotic with Achnatherum inebrians, drunken horse grass. Mycologia 107, 863-873 (2015).

43. Larriba, E. et al. Sequencing and functional analysis of the genome of a nematode egg-parasitic fungus, Pochonia chlamydosporia. Fungal Genet. Biol 65, 69-80 (2014).

44. Xiao, G. et al. Genomic perspectives on the evolution of fungal entomopathogenicity in Beauveria bassiana. Sci. Rep. 2, 483 (2012).

45. Shang, Y. et al. Divergent and convergent evolution of fungal pathogenicity. Genome Biol. Evol. 8, 1374-1387 (2016).

46. Cheeseman, K. et al. Multiple recent horizontal transfers of a large genomic region in cheese making fungi. Nat. Commun. 5, 2876 (2013).

47. Theis, T., Wedde, M., Meyer, V. \& Stahl, U. The antifungal protein from Aspergillus giganteus causes membrane permeabilization. Antimicrob. Agents Chemother. 47, 588-593 (2003).

48. Eder, J. \& Fersht, A. R. Pro-sequence-assisted protein folding. Mol. Microbiol. 16, 609-614 (1995).

49. Scott, B. et al. Functional analysis of the Epichloë festucae -perennial ryegrass symbiosis. $6^{\text {th }}$ International Symposium on Fungal Endophytes of Grasses (2007)

50. Weld, R. J., Plummer, K. M., Carpenter, M. A. \& Ridgway, H. J. Approaches to functional genomics in filamentous fungi. Cell Res. 16, 31-44 (2006)

51. Niones, J. T. \& Takemoto, D. An isolate of Epichloë festucae, an endophytic fungus of temperate grasses, has growth inhibitory activity against selected grass pathogens. J. Gen. Plant Pathol. 80, 337-347 (2014).

52. Niones, J. T. \& Takemoto, D. VibA, a homologue of a transcription factor for fungal heterokaryon incompatibility, is involved in antifungal compound production in the plant-symbiotic fungus Epichloë festucae. Eukaryot. Cell 14, 13-24 (2015).

53. Soanes, D. \& Richards, T. A. Horizontal gene transfer in eukaryotic plant pathogens. Annu. Rev. Phytopathol. 52, 583-614 (2014).

54. Qiu, H., Cai, G., Luo, J., Bhattacharya, D. \& Zhang, N. Extensive horizontal gene transfers between plant pathogenic fungi. BMC Biology 14, 41 (2016)

55. Dupont, P.-Y. \& Cox, M. P. Genomic data quality impacts automated detection of lateral gene transfer in fungi. G3 Genes Genom. Genet.22 7, 1301-1314 (2017).

56. Johnson-Cicalese, J. et al. Cross species inoculation of Chewings and strong creeping red fescues with fungal endophytes. Crop Sci 40, 1485-1489 (2000).

57. Saha, D. C., Jackson, M. A. \& Johnson-Cicalese, J. M. A rapid staining method for detection of endophytic fungi in turf and forage grasses. Phytopathology 78, 237-239 (1988).

58. Moy, M., Li, H. M., Sullivan, R., White, J. F. Jr. \& Belanger, F. C. Endophytic fungal $\beta$-1,6-glucanase expression in the infected host grass. Plant Physiol 130, 1298-1308 (2002).

59. Laemmli, U. K. Cleavage of structural proteins during the assembly of the head of bacteriophage T4. Nature 227, 680-685 (1970).

60. Moghaddam, A. \& Reinton, N. Use of polyethylene glycol for drying polyacrylamide gels to avoid cracking. SciPhu Publishing. http://sciphu.com/2008/03/use-of-polyethylene-glycol-for-drying.html (2008).

61. Ambrose, K. V. et al. Functional characterization of salicylate hydroxylase from the fungal endophyte Epichloë festucae. Sci. Rep. 5, 10939 (2015)

62. Cabral, K. M. S., Almeida, M. S., Valente, A. P., Almeida, F. C. L. \& Kurtenbach, E. Production of the active antifungal Pisum sativum defensin 1 (Psd1) in Pichia pastoris: overcoming the inefficiency of the STE13 protease. Protein Expr. Purif. 31, 115-122 (2003).

63. Waters, M. G., Evans, E. A. \& Blobel, G. Prepro $\alpha$-factor has a cleavable signal sequence. J. Biol. Chem. 263, 6209-6214 (1988).

64. Shehata, H. R., Lyons, E. M., Jordan, K. S. \& Raizada, M. N. Bacterial endophytes from wild and ancient maize are able to suppress the fungal pathogen Sclerotinia homoeocarpa. J. Appl. Microbiol. 120, 756-769 (2016).

65. Thompson, J. D., Gibson, T. J., Plewniak, F., Jeanmougin, F. \& Higgins, D. G. The CLUSTAL-X windows interface: flexible strategies for multiple sequence alignment aided by quality analysis tools. Nuc. Acids Res 25, 4876-4882 (1997).

\section{Acknowledgements}

This work was supported with funds provided by the United States Golf Association, the Rutgers Center for Turfgrass Science and the USDA National Institute of Food and Agriculture Hatch project accession number 1007068 through the New Jersey Agricultural Experiment Station, Hatch project NJ12140.

\section{Author Contributions}

Z.T., R.W., K.V.A., B.B.C., and F.C.B. conceived and designed the experiments; Z.T., R.W., and K.V.A. performed the experiments. All authors analyzed the results and wrote the manuscript.

Additional Information

Supplementary information accompanies this paper at doi:10.1038/s41598-017-06068-4 
Competing Interests: The authors declare that they have no competing interests.

Publisher's note: Springer Nature remains neutral with regard to jurisdictional claims in published maps and institutional affiliations.

(c) (i) Open Access This article is licensed under a Creative Commons Attribution 4.0 International (c) License, which permits use, sharing, adaptation, distribution and reproduction in any medium or format, as long as you give appropriate credit to the original author(s) and the source, provide a link to the Creative Commons license, and indicate if changes were made. The images or other third party material in this article are included in the article's Creative Commons license, unless indicated otherwise in a credit line to the material. If material is not included in the article's Creative Commons license and your intended use is not permitted by statutory regulation or exceeds the permitted use, you will need to obtain permission directly from the copyright holder. To view a copy of this license, visit http://creativecommons.org/licenses/by/4.0/.

(c) The Author(s) 2017 